\title{
NUMERICAL SOLUTION OF A 1-D ELASTOHYDRODYNAMIC PROBLEM IN MAGNETIC STORAGE DEVICES
}

\author{
IÑigo Arregui ${ }^{1}$, José Jesús Cendán ${ }^{1}$, Carlos Parés ${ }^{2}$ and Carlos Vázquez ${ }^{1}$
}

\begin{abstract}
In this work we present new numerical methods to simulate the mechanics of head-tape magnetic storage devices. The elastohydrodynamic problem is formulated in terms of a coupled system which is governed by a nonlinear compressible Reynolds equation for the air pressure over the head, and a rod model for the tape displacement. A fixed point algorithm between the solutions of the elastic and hydrodynamic problems is proposed. For the nonlinear Reynolds equation, a characteristics method and a duality algorithm are developed to cope with the convection dominating and nonlinear diffusion features, respectively. Furthermore, in the duality method the convergence and optimal choice of the parameters are analyzed. At each fixed point iteration, in the elastic model a complementarity formulation is required and appropriate numerical techniques are used. For the spatial discretization different finite element spaces are chosen. Finally, numerical test examples illustrate the theoretical results, as well as the good performance in the simulation of real devices.
\end{abstract}

Mathematics Subject Classification. 76A20, 76D08, 35J60, 65J15, 65N60.

Received May 10, 2007. Revised January 8, 2008.

Published online June 5, 2008.

\section{INTRODUCTION}

In magnetic tape recording, the tape is passed over a transducer, called the head, to convert electrical and magnetic signals. The mechanical behaviour of the tape is crucial to determine the quality of the signal. Thus, the strength of the signal decreases with distance between the head and the tape, while the wear on the head and the tape increases as the gap tends to zero. Therefore it is desirable to maintain an optimal and small enough flying height between the head and the tape. These arguments can be extended to other magnetic storage devices (see [6], for example). The tape movement causes a thin air film to be trapped between the tape and the head at the beginning of the reading process. Typically, a convergent-divergent gap profile arises that increases the air pressure above the atmospheric one and, consequently, the tape moves away from the head, so that the hydrodynamic load (due to the air pressure) balances the external load (due to the tape weight). The design of suitable head-type interfaces only based on experimental tests is expensive and very time consuming. So, the use of appropriate mathematical models and efficient numerical techniques is highly interesting.

\footnotetext{
Keywords and phrases. Head-tape devices, elastohydrodynamic lubrication, compressible Reynolds equation, rod model, duality methods, finite elements.

1 Depto. de Matemáticas, Universidade da Coruña, Facultad de Informática, Campus de Elviña, s/n, 15071-La Coruña, Spain. carlosv@udc.es

2 Depto. de Análisis Matemático, Universidad de Málaga, Facultad de Ciencias, Campus de Teatinos, s/n, 29080-Málaga, Spain.
} 
In flexible storage media, such as head-tape devices, any tape movement affects the air pressure and this one in turn influences the tape displacement. So, a typical elastohydrodynamic problem is posed, where the fluid pressure and the tape movement are highly related. In this thin film setting, the steady state fluid pressure is usually modelled by a first-order-slip compressible Reynolds equation (gas bearing model), where the gap function enters as an unknown coefficient to be obtained from an appropriate elastic model governing the tape displacement and deformation. The main interest is to compute the steady state separation.

The consideration of wide enough tapes (with respect to its length) allows to neglect side flow effects and leads to models where the pressure and the gap only depend on the variable in the displacement direction [6]. This is not the case when narrow tapes are considered or imperfections in the parameters of the system (such as axial tension variations) can cause asymmetries in the transverse direction to the displacement [6]. The consideration of either one or two dimensional models strongly depends on the head and tape geometries as well as on operating conditions of the device. Nevertheless, as pointed out in $[27,28]$, when the cross section of the head remains uniform along the width of the tape a one dimensional model can be used mainly to estimate the gap at the longitudinal central line where side effects are negligible. For example, also one dimensional models have been recently used in the modelling of head-tape interface in a digital linear tape drive [24].

One-dimensional head-tape models were initially proposed by Stahl et al. [23], who introduced a transient coupled problem between a first-order-slip parabolic compressible Reynolds equation for the pressure and a fourth order transient rod model for the tape deflection. The steady state was computed by iteration in the numerical solution of the time dependent problem until convergence. More precisely, in [23] a fixed point iteration between the finite differences discretized problems of both time dependent equations is developed, the nonlinear diffusive term in Reynolds equation being treated explicitly. This method has been heuristically improved in [18] by means of a different linearization of the diffusive term and a fictitious foundation stiffness in the tape equation as stabilizing technique. In [16] a spatial finite element discretization is developed on the transient problem which is posed in different unknowns, the nonlinear diffusive term being treated by a Newton method with initial guess obtained from the previous time step. In [19] a finite element technique is proposed for the 1-d model. More recently, in [26] some results concerning the finite element solution of a steady state coupled problem between a 1-d compressible Reynolds equation and a tape deflection equation are presented. Furthermore, local pressure effects due to asperities in ultra thin devices are taken into account, the discretized nonlinear system being solved by means of a Newton method. Some comparisons between numerical results and interferometric experimental measurements are provided. In [27,28], straightforward extensions of the numerical techniques in [26] to 2-d models are presented.

On the other hand, the rigorous mathematical analysis of well posed 1-d head-tape models is developed in $[13,14]$, where existence of solution is obtained by different techniques.

In the case of rigid magnetic storage devices, such as hard disks in computers, the thin gap between the head and the disk is assumed to be given, so that only the air pressure has to be obtained in a purely hydrodynamic problem (see [8] for the mathematical analysis of different Reynolds equations depending on the order of the gap). Particularly, as in the present paper, a first-order-slip compressible Reynolds equation has been considered in Jai [17], where two scale homogenization techniques are applied to deal with the presence of surface roughness. Also two numerical alternatives mainly based on fixed point and Newton techniques are proposed to solve the nonlinear Reynolds equation with oscillating coefficients and the homogenized problems. In [7], the complexity and limitations of using a Newton technique for the nonlinear diffusive term are pointed out and a combination with a continuation method is applied. More recently, in [2] we have introduced a new numerical method to solve a 1-d nonlinear compressible Reynolds equation which mainly takes advantage of writing the problem in terms of an appropriate maximal monotone operator, preventing from the use of general purpose Newton methods and the complexity and limitations associated to them. In all previous papers the convergence of the involved numerical methods is not mathematically analyzed.

In the present paper, we propose an efficient numerical method to solve the one dimensional elastohydrodynamic problem governed by a first-order-slip compressible Reynolds equation and a clamped rod model. For this purpose, a fixed point iteration between the solution of the hydrodynamic and elastic subproblems is carried out. 


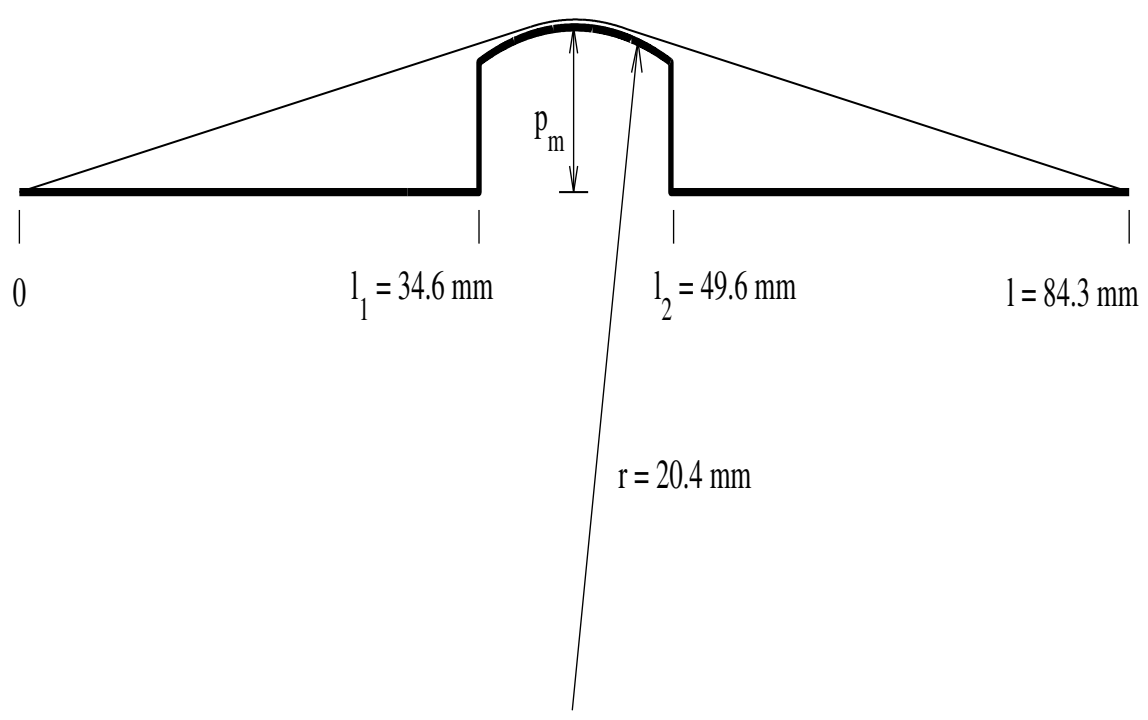

Figure 1. Geometry of the magnetic device.

For the numerical solution of the hydrodynamic part, first a characteristics method (adapted to steady state problems) is developed to cope with the convection dominated feature that arises when considering real data sets. Secondly, a new version of a duality method is proposed to deal with the nonlinear diffusive term appearing in Reynolds equation. In [2] a classical constant parameter version of this duality method has been applied. In the present paper, both the constant and functional parameter versions of the method are applied in the head-tape elastohydrodynamic setting. Furthermore, for a particular discretized problem in the constant parameters case, the convergence is stated by using mathematical tools related to maximal monotone operators, thus extending previous results on the method to a new type of functional equations. Also, the optimal choice for the functional parameters version is obtained, thus improving the performance of the method. Both results are illustrated with numerical examples. For the clamped rod model, an obstacle problem formulation is required at each step of the fixed point algorithm as the tape position is not guaranteed to be over the head. Next, different appropriate classical numerical techniques (Hermite finite elements combined with projection or Uzawa algorithms) are applied to the fourth order elliptic variational inequality.

Although the theoretical results are partial and limited to 1-d models, the extension to 2-d models of its implementation is straightforward and has already been developed in [3], where a new Reynolds-Koiter model is proposed, mathematically analyzed and numerically solved.

Thus, the plan of the paper is as follows. In Section 2, we present the coupled mathematical model, jointly with the fixed point algorithm. In Section 3, we describe the numerical methods concerning the hydrodynamic problem and we state the theoretical results about the convergence (constant parameter case) and the optimal choice (functional parameter case). Also we present some test examples to illustrate these results. In Section 4, we briefly describe the numerical methods for the elastic subproblem. In Section 5, we show some results obtained for a real data coupled problem.

\section{The COUpled MATHEmatical model. A FiXed POINT ALGorithm}

Figure 1 sketches the schematic of the one dimensional head-media interaction system, showing the tape path over the head and the gap between the head and the storage media. It is a free span between two supporting tape guides which determine the length of the parameterization interval, $l$, of the tape position. Thus, the ends of the tape are placed at $x=0$ and $x=l$, and the edges of the head are located at $x=l_{1}$ and $x=l_{2}$, respectively. 


\section{Tape}

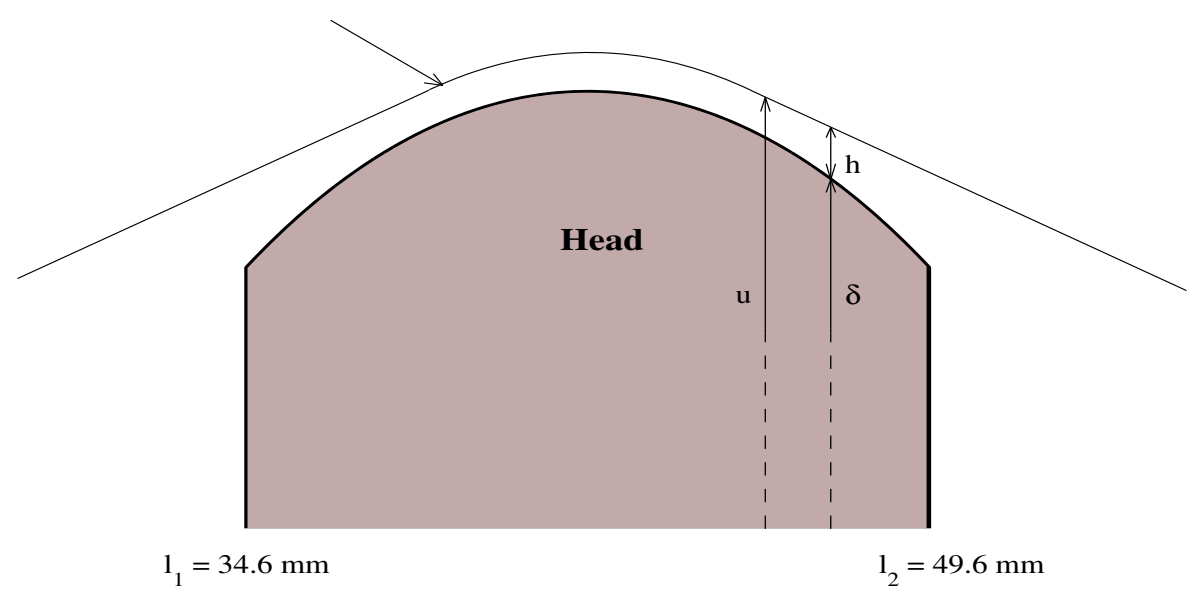

FiguRE 2. Zoom of the central part of the device (head-tape contact).

Once the steady state is achieved, we assume that the velocity of the tape, $V$, is constant in the $x$ direction. Moreover, air will be considered a perfect gas (with density proportional to pressure) in Newtonian and laminar regime, inertial forces and stress effects are negligible, and constant viscosity and temperature will be assumed. Finally, for the elastic tape we assume small displacements and we impose clamped boundary conditions at both ends located at the tape guides positions.

In this setting, we consider a fourth order linear rod model to describe the tape displacement, $u$, and a nonlinear compressible Reynolds equation to model the air pressure distribution, $p$, inside the thin film between the head and the tape. Thus, the coupled problem to find $p$ and $u$ is governed by the following set of equations (see Friedman [12]):

$$
\begin{array}{ll}
6 V \mu \frac{\mathrm{d}}{\mathrm{d} x}(p h)-6 \lambda p_{a} \frac{\mathrm{d}}{\mathrm{d} x}\left(h^{2} \frac{\mathrm{d} p}{\mathrm{~d} x}\right)-\frac{\mathrm{d}}{\mathrm{d} x}\left(h^{3} p \frac{\mathrm{d} p}{\mathrm{~d} x}\right)=0 & \text { in }\left(l_{1}, l_{2}\right), \\
-\left(T-\rho V^{2}\right) \frac{\mathrm{d}^{2} u}{\mathrm{~d} x^{2}}+E I \frac{\mathrm{d}^{4} u}{\mathrm{~d} x^{4}}=\left(p-p_{a}\right) \chi_{\left[l_{1}, l_{2}\right]} & \text { in }(0, l), \\
h=u-\bar{\delta} & \text { in }(0, l), \\
p\left(l_{1}\right)=p\left(l_{2}\right)=p_{a}, & \\
u(0)=u(l)=\frac{\mathrm{d} u}{\mathrm{~d} x}(0)=\frac{\mathrm{d} u}{\mathrm{~d} x}(l)=0, &
\end{array}
$$

where $V$ is the velocity of the tape, $\lambda$ is the molecular mean free path of the air, $p_{a}$ is the ambient pressure and $\mu$ is the viscosity of the air. Moreover, $T, \rho, E$ and $I$ represent tension, density, Young modulus and inertia moment of the tape, respectively. The notation $\chi_{C}$ stands for the characteristic function of the set $C$.

Thus, for a given gap, $h$, equation (2.1) is a one dimensional compressible Reynolds equation that governs the air pressure just over the head. On the other hand, for a known pressure distribution over the head with atmospheric value away from the head, equation (2.2) defines the position of the tape. The coupled feature of the problem arises from two main facts: the presence of the gap, $h=u-\bar{\delta}$, in equation (2.1), where the function $\bar{\delta}$ defines the geometry of the head, and the presence of the pressure in equation (2.2), acting as a normal force to the tape. 
Notice that the nonlinear equation (2.1) has three terms: a first advective term, a second linear diffusive one and a third nonlinear diffusive term. So, the hydrodynamic problem is governed by a convection-diffusion equation with nonlinear diffusion. Furthermore, in real data applications, convection dominates diffusion.

Dirichlet boundary conditions at both ends of the head impose an atmospheric pressure by means of equation (2.4), while clamped boundary conditions on the displacement are considered at both extremities of the tape by equation (2.5).

Concerning other data appearing in the coupled model, we consider a reading head with upper circular profile given by the function [12]:

$$
\bar{\delta}(x)=b+\sqrt{r^{2}-\left(x-\frac{1}{2}\left(l_{1}+l_{2}\right)\right)^{2}}, \quad x \in\left(l_{1}, l_{2}\right),
$$

where $r$ is the head radius and $b=p_{m}-r, p_{m}$ being termed as the maximum head penetration. The function $\bar{\delta}$ is understood to be extended by zero outside the head limits, when necessary. Typical values of the different parameters involved are (see [18], for example):

$$
\begin{array}{lll}
V=2.54 \mathrm{~m} \cdot \mathrm{s}^{-1}, & r=0.0204 \mathrm{~m}, & p_{m}=0.00635 \mathrm{~m}, \\
l_{1}=0.0347 \mathrm{~m}, & l_{2}=0.0497 \mathrm{~m}, & l=0.0843 \mathrm{~m}, \\
\mu=1.81 \times 10^{-5} \mathrm{~kg} \cdot \mathrm{s}^{-1} \cdot \mathrm{m}^{-1}, & \lambda=6.35 \times 10^{-8} \mathrm{~m}, & p_{a}=84100 \mathrm{~N} \cdot \mathrm{m}^{-2}, \\
T=277 \mathrm{~N} \cdot \mathrm{m}^{-1}, & \rho=0.0207 \mathrm{~kg} \cdot \mathrm{m}^{-2}, & E I=1.52 \times 10^{-5} \mathrm{~N} \cdot \mathrm{m} .
\end{array}
$$

The mathematical analysis of a very close model to the previous one has been developed in [13]. In this analysis, the following change of unknowns and variables is introduced:

$$
X=100 x, \quad P=p / p_{a}, \quad U=10^{6} u, \quad H=10^{6} h, \quad \delta=10^{6} \bar{\delta},
$$

so that the following system of dimensionless equations is obtained:

$$
\begin{array}{ll}
\frac{\mathrm{d}}{\mathrm{d} X}(P H)-\varepsilon \frac{\mathrm{d}}{\mathrm{d} X}\left(\alpha H^{2} \frac{\mathrm{d} P}{\mathrm{~d} X}+\beta H^{3} P \frac{\mathrm{d} P}{\mathrm{~d} X}\right)=0 & \text { in }\left(L_{1}, L_{2}\right), \\
-\frac{\mathrm{d}^{2} U}{\mathrm{~d} X^{2}}+\eta \frac{\mathrm{d}^{4} U}{\mathrm{~d} X^{4}}=K(P-1) \chi_{\left[L_{1}, L_{2}\right]} & \text { in }(0, L), \\
H=U-\delta & \text { in }(0, L), \\
P\left(L_{1}\right)=P\left(L_{2}\right)=1, & \\
U(0)=U(L)=\frac{\mathrm{d} U}{\mathrm{~d} X}(0)=\frac{\mathrm{d} U}{\mathrm{~d} X}(L)=0, &
\end{array}
$$

where the involved coefficients are given by

$$
\alpha=10^{-4} \frac{\lambda p_{a}}{\varepsilon \mu V}, \quad \beta=10^{-10} \frac{p_{a}}{6 \varepsilon \mu V}, \quad \eta=10^{4} \frac{E I}{T-\rho V^{2}}, \quad K=10^{2} \frac{p_{a}}{T-\rho V^{2}} .
$$

Notice that the scaling (2.7) allows to obtain in [13] the existence of solution, first analyzing the limit case $\epsilon=\eta=0$, and points out the convection dominating feature of the convective-nonlinear diffusive problem for the air pressure. This aspect motivates the use of appropriate numerical methods to deal with convection dominated problems as, for example, characteristics techniques proposed in the present paper. More precisely, in [13] the following result is stated: 
Theorem 2.1. If $\delta \in \mathcal{C}^{2}\left(L_{1}, L_{2}\right)$ verifies that $\delta>0, \delta^{\prime \prime}<0, \delta\left(L_{1}\right)<\delta^{\prime}\left(L_{1}\right) L_{1}$ and $\delta\left(L_{2}\right)<\delta^{\prime}\left(L_{2}\right)\left(L_{2}-L\right)$, then there exist positive constants $\varepsilon^{+}$and $\eta^{+}$such that if $0<\varepsilon<\varepsilon^{+}$and $0<\eta<\eta^{+}$, the system of equations (2.8)(2.12) has a classical solution $(P, H, U)$ with $P \in W^{1, \infty}\left(L_{1}, L_{2}\right), H \in W^{4, \infty}(0, L)$ and $P>0, H>0$ in $\left[L_{1}, L_{2}\right]$.

This result is stated by means of a shooting method, after analyzing the limit case $\eta=\varepsilon=0$. This technique is replaced by a comparison argument involving sub and supersolutions in [14], where the result is extended to the case with slots in the head surface (giving rise to discontinuities in the function which defines the head upper profile).

Once we have stated the coupled model and recalled the mathematical analysis results, in order to compute an approximated solution we propose a fixed point iterative scheme, which essentially uncouples the hydrodynamic and elastic parts of the problem. Thus, as in other related elastohydrodynamic problems (see $[1,10,11,25]$, for example), we sequentially solve the compressible Reynolds equation and the rod model until fixed point convergence.

Before stating the algorithm, we notice that, although the existence result in [13] guarantees that the position of the tape is over the head, we cannot guarantee this property for the solution of the elastic problem at each step of fixed point algorithm. This is the reason why we replace the elastic problem by a linear complementarity formulation, where the obstacle condition imposes the tape to be placed over the head. Near convergence, the region where the tape touches the obstacle disappears. After this important remark, the proposed algorithm can be written as follows:

- Step 0: initial value $P^{0}$.

- Step $n+1$ :

- Elastic part: For $P^{n}$ given, we obtain $U^{n+1}$ such that:

$$
\left(\mathcal{P}_{E}^{n+1}\right) \begin{cases}-\frac{\mathrm{d}^{2} U^{n+1}}{\mathrm{~d} X^{2}}+\eta \frac{\mathrm{d}^{4} U^{n+1}}{\mathrm{~d} X^{4}} \geq K\left(P^{n}-1\right) \chi_{\left[L_{1}, L_{2}\right]}, & X \in(0, L) \\ U^{n+1} \geq \delta, & X \in(0, L) \\ {\left[-\frac{\mathrm{d}^{2} U^{n+1}}{\mathrm{~d} X^{2}}+\eta \frac{\mathrm{d}^{4} U^{n+1}}{\mathrm{~d} X^{4}}-K\left(P^{n}-1\right) \chi_{\left[L_{1}, L_{2}\right]}\right] \cdot\left(U^{n+1}-\delta\right)=0,} & X \in(0, L) \\ U^{n+1}(0)=U^{n+1}(L)=\frac{\mathrm{d} U^{n+1}}{\mathrm{~d} X}(0)=\frac{\mathrm{d} U^{n+1}}{\mathrm{~d} X}(L)=0 . & \end{cases}
$$

- Hydrodynamic part: For $H^{n+1}=U^{n+1}-\delta$ given, we obtain $P^{n+1}$ such that:

$$
\left(\mathcal{P}_{H}^{n+1}\right)\left\{\begin{array}{r}
\frac{\mathrm{d}}{\mathrm{d} X}\left(P^{n+1} H^{n+1}\right)-\varepsilon \alpha \frac{\mathrm{d}}{\mathrm{d} X}\left(\left(H^{n+1}\right)^{2} \frac{\mathrm{d} P^{n+1}}{\mathrm{~d} X}\right) \\
-\varepsilon \beta \frac{\mathrm{d}}{\mathrm{d} X}\left(\left(H^{n+1}\right)^{3} P^{n+1} \frac{\mathrm{d} P^{n+1}}{\mathrm{~d} X}\right)=0, \quad X \in\left(L_{1}, L_{2}\right) \\
P^{n+1}\left(L_{1}\right)=P^{n+1}\left(L_{2}\right)=1
\end{array}\right.
$$

For simplicity, we have taken $\varepsilon=1$ in the rest of the paper.

\section{NUMERICAL SOLUTION OF THE HYDRODYNAMIC MODEL}

Several difficulties arise when addressing the numerical solution of problem $\left(\mathcal{P}_{H}^{n+1}\right)$. First, the dimensionless compressible Reynolds equation presents a nonlinear diffusive term; secondly, in real applications $\alpha=O\left(10^{-2}\right)$ and $\beta=O\left(10^{-2}\right)$, so that the advection effects are larger than the diffusion ones (convection 
dominating feature). In this section, we focus on the numerical solution of this problem and we propose some specific techniques to overcome these difficulties. In [23] the finite differences discretization of the evolutive problems requires extremely small time steps and the nonlinear diffusive term is treated explicitly (with coefficient $\left(H^{n+1}\right)^{3} P^{n}$ replacing $\left.\left(H^{n+1}\right)^{3} P^{n+1}\right)$. Notice that the steady state is obtained as the converged solution of the transient problems. In [18], the nonlinear term is written in the form $\partial\left(P^{n+1}\right)^{2} / \partial X$ and it is linearized by replacing $\left(P^{n+1}\right)^{2}$ with $2 P^{n+1} P^{n}-\left(P^{n}\right)^{2}$. More recently, in [17] two alternatives are proposed: a fixed point iteration where the diffusion coefficient is explicitly taken, and a LPDEM method mainly based on a Kirchhoff-like change of unknown which linearizes the diffusive term and uses a Newton method to deal with the resulting nonlinear convective term. More recently, in [7] a Newton method is proposed to deal with the nonlinear diffusive term in a compressible homogenized Reynolds equation, although the authors point out that the convergence strongly depends on the initial guess close to the solution. For this reason, they also combine it with a continuation method. In all previously proposed algorithms a convergence analysis is not provided and some of them are heuristically designed to simulate the devices.

In the present paper, a characteristics algorithm is proposed to deal with the convection dominating feature and a duality method is used to solve the particular nonlinearity appearing in the diffusive term. Furthermore, for this duality algorithm a convergence result is stated for the constant parameter case and the functional parameters are optimized in order to reduce the number of iterations needed to attain the convergence.

\subsection{Numerical methods}

First, as throughout this section we describe the numerical solution of $\left(\mathcal{P}_{H}^{n+1}\right)$, we drop the index $n+1$ for simplicity (so, we note $P=P^{n+1}$ and $H=H^{n+1}$ ). Next, in order to use a finite element method, let us consider the following variational formulation of the hydrodynamic problem $\left(\mathcal{P}_{H}^{n+1}\right)$ :

Find $P \in V_{1}$ such that:

$$
\int_{L_{1}}^{L_{2}} \frac{\mathrm{d}(P H)}{\mathrm{d} X} \varphi \mathrm{d} X+\int_{L_{1}}^{L_{2}}\left(\alpha H^{2}+\beta H^{3} P\right) \frac{\mathrm{d} P}{\mathrm{~d} X} \frac{\mathrm{d} \varphi}{\mathrm{d} X} \mathrm{~d} X=0, \quad \forall \varphi \in V_{0},
$$

where the functional sets are:

$$
V_{0}=H_{0}^{1}\left(L_{1}, L_{2}\right) \quad \text { and } \quad V_{1}=\left\{\varphi \in H^{1}\left(L_{1}, L_{2}\right) / \varphi\left(L_{1}\right)=\varphi\left(L_{2}\right)=1\right\} .
$$

Next, taking into account the dominating convection feature in (3.1), following [2] we propose to adapt a characteristics technique for steady state problems. This method introduces an artificial dependence on a time variable, $t$, so that:

$$
\bar{P}(x, t)=P(x) \quad \bar{H}(x, t)=H(x) .
$$

If we assume an artificial velocity field $v(x)=1$, we have the following identities for the total derivative:

$$
\frac{D(\bar{P} \bar{H})}{D t}(x, t)=\frac{\partial(\bar{P} \bar{H})}{\partial t}(x, t)+v(x) \frac{\partial(\bar{P} \bar{H})}{\partial x}(x, t)=\frac{\mathrm{d}(P H)}{\mathrm{d} x}(x),
$$

where $D / D t$ represents the material derivative along the characteristic line:

$$
\tau \longrightarrow \chi(x, t ; \tau),
$$

which is the unique solution of the final value problem:

$$
\left\{\begin{array}{l}
\frac{\mathrm{d} \chi}{\mathrm{d} \tau}(x, t ; \tau)=v(\chi(x, t ; \tau))=1, \\
\chi(x, t ; t)=x
\end{array}\right.
$$


In order to discretize the total derivative, for an artificial time step $k$ we denote by $\chi^{k}(x)=\chi(x, t ; t-k)$ the position of a material particle in the instant $t-k$. Notice that, taking into account the value of artificial velocity, $\chi(\tau)=x+\tau-t$ is straightforwardly obtained.

So, we approximate the material derivative by a first order quotient:

$$
\frac{D(\bar{P} \bar{H})}{D t}(x, t) \approx \frac{(\bar{P} \bar{H})(x, t)-(\bar{P} \bar{H})\left(\chi^{k}(x), t-k\right)}{k}=\frac{(P H)(x)-(P H)(x-k)}{k},
$$

and, after substitution in (3.1), we propose to obtain the unknown, $P$, as the limit of the sequence $\left\{P_{m}\right\}$, which is defined by the following algorithm:

For $m \geq 0$ and $P_{m}$ given, we search $P_{m+1} \in V_{1}$ such that:

$$
\int_{L_{1}}^{L_{2}} P_{m+1} H \varphi \mathrm{d} X+k \int_{L_{1}}^{L_{2}}\left(\alpha H^{2}+\beta H^{3} P_{m+1}\right) \frac{\mathrm{d} P_{m+1}}{\mathrm{~d} X} \frac{\mathrm{d} \varphi}{\mathrm{d} X} \mathrm{~d} X=\int_{L_{1}}^{L_{2}}\left(\left(P_{m} H\right) \circ \chi^{k}\right) \varphi \mathrm{d} X, \quad \forall \varphi \in V_{0},
$$

where $k$ is the artificial time step, and $\chi^{k}(X)=X-k$ is related to the characteristics method. Notice that (3.2) is a nonlinear diffusion problem.

Next, to solve the nonlinear problem (3.2), we apply a duality algorithm, which is an extension of the one proposed in [5] to solve variational inequalities. For this purpose, we first consider the maximal monotone operator $G$ given by:

$$
G(P)= \begin{cases}0, & \text { if } P<0, \\ P^{2}, & \text { if } P \geq 0,\end{cases}
$$

so that the variational equation $(3.2)$ can be written as:

$$
\int_{L_{1}}^{L_{2}} P_{m+1} H \varphi \mathrm{d} X+k \int_{L_{1}}^{L_{2}}\left(\alpha H^{2} \frac{\mathrm{d} P_{m+1}}{\mathrm{~d} X}+\frac{\beta H^{3}}{2} \frac{\mathrm{d}\left(G\left(P_{m+1}\right)\right)}{\mathrm{d} X}\right) \frac{\mathrm{d} \varphi}{\mathrm{d} X} \mathrm{~d} X=\int_{L_{1}}^{L_{2}}\left(\left(P_{m} H\right) \circ \chi^{k}\right) \varphi \mathrm{d} X, \quad \forall \varphi \in V_{0} .
$$

Next, following [20], for a given function $\omega>0$, such that $\omega \in W^{1, \infty}\left(L_{1}, L_{2}\right)$ and $1 / \omega \in W^{1, \infty}\left(L_{1}, L_{2}\right)$, we introduce the new unknown $\theta_{m+1}=G\left(P_{m+1}\right)-\omega P_{m+1}$. So, we search $\left(P_{m+1}, \theta_{m+1}\right)$ verifying the still nonlinear problem:

$$
\begin{aligned}
\int_{L_{1}}^{L_{2}} P_{m+1} H \varphi \mathrm{d} X+k \int_{L_{1}}^{L_{2}}\left(\alpha H^{2}+\frac{\beta \omega}{2} H^{3}\right) \frac{\mathrm{d} P_{m+1}}{\mathrm{~d} X} \frac{\mathrm{d} \varphi}{\mathrm{d} X} \mathrm{~d} X \\
+\frac{k \beta}{2} \int_{L_{1}}^{L_{2}} H^{3} \frac{\mathrm{d} \omega}{\mathrm{d} X} P_{m+1} \frac{\mathrm{d} \varphi}{\mathrm{d} X} \mathrm{~d} X=\int_{L_{1}}^{L_{2}}\left(\left(P_{m} H\right) \circ \chi^{k}\right) \varphi \mathrm{d} X \\
\quad-\frac{k \beta}{2} \int_{L_{1}}^{L_{2}} H^{3} \frac{\mathrm{d} \theta_{m+1}}{\mathrm{~d} X} \frac{\mathrm{d} \varphi}{\mathrm{d} X} \mathrm{~d} X, \quad \forall \varphi \in V_{0} \\
\theta_{m+1}=G\left(P_{m+1}\right)-\omega P_{m+1} .
\end{aligned}
$$

In [5], the function $\omega$ is taken as constant and usually known as Bermúdez-Moreno parameter; this classical choice is refered hereafter as the constant parameter version. More recently, the convergence results have been extended in [20] to the choice of $\omega$ as a function, hereafter refered as the variable or functional parameter version. Particularly, we can use the lemma for the functional parameter version [20] and replace (3.5) by

$$
\theta_{m+1}=G_{1 / 2 \omega}^{\omega}\left(P_{m+1}+\frac{1}{2 \omega} \theta_{m+1}\right)
$$


where $G_{1 / 2 \omega}^{\omega}$ is the Yosida approximation of $G-\omega I$ with parameter $1 / 2 \omega$, the definition of the Yosida approximation of parameter $\lambda$ being given by:

$$
G_{\lambda}^{\omega}(z)=\frac{1}{\lambda}\left(I-\left(\frac{1}{(1-\lambda \omega)} I+\lambda G\right)^{-1}\right)(z) .
$$

In the previous expressions $I$ denotes the identity operator. Finally, in order to overcome the nonlinearity in (3.6), we propose the following fixed-point algorithm that iterates between equations (3.4) and (3.6):

- For $\theta_{m+1}^{\ell}$ known, find $P_{m+1}^{\ell+1}$ verifying the linear problem:

$$
\begin{aligned}
\int_{L_{1}}^{L_{2}} P_{m+1}^{\ell+1} H \varphi \mathrm{d} X+k \int_{L_{1}}^{L_{2}}\left(\alpha H^{2}+\frac{\beta \omega}{2} H^{3}\right) \frac{\mathrm{d} P_{m+1}^{\ell+1}}{\mathrm{~d} X} \frac{\mathrm{d} \varphi}{\mathrm{d} X} \mathrm{~d} X \\
+\frac{k \beta}{2} \int_{L_{1}}^{L_{2}} H^{3} \frac{\mathrm{d} \omega}{\mathrm{d} X} P_{m+1}^{\ell+1} \frac{\mathrm{d} \varphi}{\mathrm{d} X} \mathrm{~d} X=\int_{L_{1}}^{L_{2}}\left(\left(P_{m} H\right) \circ \chi^{k}\right) \varphi \mathrm{d} X \\
\quad-\frac{k \beta}{2} \int_{L_{1}}^{L_{2}} H^{3} \frac{\mathrm{d} \theta_{m+1}^{\ell}}{\mathrm{d} X} \frac{\mathrm{d} \varphi}{\mathrm{d} X} \mathrm{~d} X, \quad \forall \varphi \in V_{0} .
\end{aligned}
$$

- For $P_{m+1}^{\ell+1}$ known, $\theta_{m+1}^{\ell+1}$ is updated by:

$$
\theta_{m+1}^{\ell+1}=G_{1 / 2 \omega}^{\omega}\left(P_{m+1}^{\ell+1}+\frac{1}{2 \omega} \theta_{m+1}^{\ell}\right)
$$

Lagrange piecewise linear finite elements are proposed for the spatial discretization of (3.7).

\subsection{Functional framework}

In order to state some theoretical results concerning the convergence and optimal choice of parameters, we introduce an abstract mathematical framework. Thus, let us consider the Hilbert spaces $E=L^{2}\left(L_{1}, L_{2}\right)=E^{\prime}$ and $V=H^{1}\left(L_{1}, L_{2}\right)$. Let be:

- $A: V \longrightarrow V^{\prime}$ the operator given by:

$$
A \psi=H \psi-\alpha k \frac{\mathrm{d}}{\mathrm{d} X}\left(H^{2} \frac{\mathrm{d} \psi}{\mathrm{d} X}\right), \quad \forall \psi \in V
$$

- $a: V \times V \longrightarrow \mathbb{R}$ the symmetric coercive bilinear form, associated to the operator $A$, given by:

$$
a(\psi, \varphi)=<A \psi, \varphi>, \quad \forall \psi, \varphi \in V
$$

- $B: E \longrightarrow V^{\prime}$ the operator given by:

$$
<B w, \varphi>=\int_{L_{1}}^{L_{2}} H^{3 / 2} w \frac{\mathrm{d} \varphi}{\mathrm{d} X} \mathrm{~d} X, \quad \forall w \in E, \quad \forall \varphi \in V
$$

- $G: V \longrightarrow V$, the maximal monotone operator given by:

$$
G(\varphi)(X)= \begin{cases}(\varphi(X))^{2}, & \text { if } \varphi(X) \geq 0 \\ 0, & \text { if } \varphi(X) \leq 0\end{cases}
$$

$G$ is well posed, due to the inclusion $V \subset L^{\infty}\left(L_{1}, L_{2}\right)$; 
- $f \in V^{\prime}$, given by:

$$
<f, \varphi>=\int_{L_{1}}^{L_{2}}\left(\left(P_{m} H\right) \circ \chi^{k}\right) \varphi \mathrm{d} X, \quad \forall \varphi \in V .
$$

In the previous abstract frame, the hydrodynamic problem of finding $P_{m+1} \in V_{1}$ solution of (3.3) is equivalent to find $y \in V_{1}$ such that:

$$
A y+c\left(B B^{*}\right)(G(y))=f,
$$

where $c=k \beta / 2$.

Thus, for $\omega \in W^{1, \infty}\left(L_{1}, L_{2}\right)$ such that $1 / \omega \in W^{1, \infty}\left(L_{1}, L_{2}\right)$, we introduce $\theta=G(y)-\omega y \in V$ and pose the fixed-point algorithm:

$$
\begin{aligned}
A y^{\ell+1}+c\left(B B^{*}\right)\left(\omega y^{\ell+1}\right) & =f-c\left(B B^{*}\right)\left(\theta^{\ell}\right) \\
\theta^{\ell+1} & =G_{1 / 2 \omega}^{\omega}\left(y^{\ell+1}+\frac{1}{2 \omega} \theta^{\ell}\right) .
\end{aligned}
$$

Remark 3.1. This duality method has never been analyzed when applied to functional equations in the form of (3.9). More precisely, the constant parameter version of the duality method has been first introduced in [5] for solving elliptic variational inequalities, so that they can be rewritten in the form:

$$
f \in L y+c M G\left(M^{*} y\right),
$$

where $L$ is a monotone operator, $M$ is an appropriately chosen operator and $G$ is a multivalued subdifferential operator. In this setting, a convergence result is stated in [5]. Also, the convergence for constant $\omega$ is obtained in [4] for the equation

$$
f \in L y+\sum_{i=1}^{m}\left(c_{i} M_{i} G_{i}\left(\Lambda_{E_{i}}^{-1} M_{i}^{*} y\right)\right)
$$

where $\mathrm{L}$ is a linear bounded operator, index $i$ corresponds to different multivalued maximal monotone operators $G_{i}$ and $\Lambda_{E_{i}}$ denotes the canonical isomorphism between $E_{i}$ and its dual space. The analysis of equation (3.13) is motivated by a gas flux modelling problem. In the constant parameter case, the performance of the algorithm strongly depends on the choice of the parameter $\omega$. The optimal choice of constant parameter has been treated in [21] for equations like (3.13) with $m=1$. In this same framework, the functional parameters case is analyzed in [20]. Notice that equation (3.9), which is motivated by the particular nonlinear diffusive term in the compressible Reynolds model, remains out of the scope of previous works. Therefore, next results concerning the convergence of the constant parameter version and optimal choice for the functional parameter case are the first ones for the functional equation (3.9).

\subsection{Convergence result for constant parameters}

In this section we state the convergence of the algorithm with constant parameters for the case $H=1$, when it is applied to the piecewise linear finite elements discretized problem. For this purpose, we introduce the spaces:

$$
\begin{aligned}
V_{h} & =\left\{\varphi_{h} \in \mathcal{C}^{0}\left(L_{1}, L_{2}\right) /\left.\varphi_{h}\right|_{K} \in P_{1}, \quad \forall K \in \tau_{h}\right\}, \\
V_{1 h} & =\left\{\varphi_{h} \in V_{h} / \varphi_{h}\left(L_{1}\right)=\varphi_{h}\left(L_{2}\right)=1\right\}, \\
V_{0 h} & =\left\{\varphi_{h} \in V_{h} / \varphi_{h}\left(L_{1}\right)=\varphi_{h}\left(L_{2}\right)=0\right\},
\end{aligned}
$$

where $\tau_{h}$ denotes a uniform finite elements mesh with $N_{h}$ nodes. Then, the nonlinear discretized problem is posed as follows: 
Find $y_{h} \in V_{1 h}$ such that:

$$
\begin{aligned}
A y_{h}+c \omega\left(B B^{*}\right) y_{h} & =f-c\left(B B^{*}\right) \theta_{h} \\
\theta_{h} & =G_{1 / 2 \omega}^{\omega}\left(y_{h}+\frac{\theta_{h}}{2 \omega}\right),
\end{aligned}
$$

and the $(\ell+1)$-th step of the fixed point algorithm to solve the previous problem can be written as follows:

For $\theta_{h}^{\ell}$ given, find $y_{h}^{\ell+1}$ and $\theta_{h}^{\ell+1}$ such that:

$$
\begin{aligned}
A y_{h}^{\ell+1}+c \omega\left(B B^{*}\right) y_{h}^{\ell+1} & =f-c\left(B B^{*}\right) \theta_{h}^{\ell} \\
\theta_{h}^{\ell+1} & =G_{1 / 2 \omega}^{\omega}\left(y_{h}^{\ell+1}+\frac{\theta_{h}^{\ell}}{2 \omega}\right) .
\end{aligned}
$$

If we particularize for the case $H=1$, equation (3.16) admits the variational formulation:

$$
a\left(y_{h}^{\ell+1}, \varphi_{h}\right)+c \omega\left(\frac{\mathrm{d} y_{h}^{\ell+1}}{\mathrm{~d} X}, \frac{\mathrm{d} \varphi_{h}}{\mathrm{~d} X}\right)=\left(f, \varphi_{h}\right)-c\left(\frac{\mathrm{d} \theta_{h}^{\ell}}{\mathrm{d} X}, \frac{\mathrm{d} \varphi_{h}}{\mathrm{~d} X}\right), \quad \forall \varphi_{h} \in V_{0 h}
$$

where we use the notation $(\cdot, \cdot)$ for the usual inner product in $L^{2}\left(L_{1}, L_{2}\right)$.

Next, we prove that the sequence $\left\{y_{h}^{\ell}\right\}$ tends to $y_{h}$ as $\ell$ goes to infinity, the function $y_{h}$ being characterized as the solution of the problem:

$$
\begin{aligned}
a\left(y_{h}, \varphi_{h}\right)+c \omega\left(\frac{\mathrm{d} y_{h}}{\mathrm{~d} X}, \frac{\mathrm{d} \varphi_{h}}{\mathrm{~d} X}\right) & =\left(f, \varphi_{h}\right)-c\left(\frac{\mathrm{d} \theta_{h}}{\mathrm{~d} X}, \frac{\mathrm{d} \varphi_{h}}{\mathrm{~d} X}\right), \quad \forall \varphi_{h} \in V_{0 h} \\
\theta_{h} & =G_{1 / 2 \omega}^{\omega}\left(y_{h}+\frac{1}{2 \omega} \theta_{h}\right) .
\end{aligned}
$$

For this purpose, we first subtract equations (3.19) and (3.18), and we choose $\varphi_{h}=y_{h}-y_{h}^{\ell+1}$, to obtain:

$$
\begin{aligned}
& a\left(y_{h}-y_{h}^{\ell+1}, y_{h}-y_{h}^{\ell+1}\right)+c\left(\frac{\mathrm{d}}{\mathrm{d} X}\left(y_{h}-y_{h}^{\ell+1}\right), \frac{\mathrm{d}}{\mathrm{d} X}\left(y_{h}-y_{h}^{\ell+1}\right)\right) \\
&+c\left(\frac{\mathrm{d}}{\mathrm{d} X}\left(\theta_{h}-\theta_{h}^{\ell}\right), \frac{\mathrm{d}}{\mathrm{d} X}\left(y_{h}-y_{h}^{\ell+1}\right)\right)=0 .
\end{aligned}
$$

Next, we consider the basis $\left\{\psi_{i}\right\}$ of $V_{0 h}$, which is orthonormal with respect to the usual product in $L^{2}\left(L_{1}, L_{2}\right)$, and is formed by the eigenfunctions of the discretized operator of the second derivative with homogeneous Dirichlet boundary conditions, i.e. we have the property:

$$
\left(\frac{\mathrm{d} \psi_{i}}{\mathrm{~d} X}, \frac{\mathrm{d} \psi_{j}}{\mathrm{~d} X}\right)=\mu_{i} \delta_{i j}, \quad 1 \leq i, j \leq N_{h}
$$

where $\left\{\mu_{i}\right\}$ is the spectrum of the operator, sorted out in the form $\mu_{1} \leq \mu_{2} \leq \ldots \leq \mu_{N_{h}}$.

So, in terms of the coordinates associated to the previous basis, we have

$$
y_{h}=\sum_{i=1}^{N_{h}} y_{i} \psi_{i}, \quad y_{h}^{\ell+1}=\sum_{i=1}^{N_{h}} y_{i}^{\ell+1} \psi_{i}, \quad \theta_{h}=\sum_{i=1}^{N_{h}} \theta_{i} \psi_{i}, \quad \theta_{h}^{\ell+1}=\sum_{i=1}^{N_{h}} \theta_{i}^{\ell+1} \psi_{i} .
$$


On the other hand, for the choice $\varphi_{h}=\psi_{i}$, the difference between (3.19) and (3.18) leads to

$$
a\left(y_{h}-y_{h}^{\ell+1}, \psi_{i}\right)+c \omega\left(\frac{\mathrm{d}}{\mathrm{d} X}\left(y_{h}-y_{h}^{\ell+1}\right), \frac{\mathrm{d}}{\mathrm{d} X} \psi_{i}\right)+c\left(\frac{\mathrm{d}}{\mathrm{d} X}\left(\theta_{h}-\theta_{h}^{\ell}\right), \frac{\mathrm{d}}{\mathrm{d} X} \psi_{i}\right)=0,1 \leq i \leq N_{h} .
$$

Now, using the expressions in (3.22) and the equations (3.23), from easy computations we get:

$$
c\left(\theta_{i}-\theta_{i}^{\ell}\right) \mu_{i}=-a\left(y_{h}-y_{h}^{\ell+1}, \psi_{i}\right)-c \omega\left(y_{i}-y_{i}^{\ell+1}\right) \mu_{i}, \quad 1 \leq i \leq N_{h} .
$$

Next, we obtain a bound for the term $\left(\theta_{h}-\theta_{h}^{\ell}, y_{h}-y_{h}^{\ell+1}\right)$. For this, we first consider the identities:

$$
\begin{aligned}
\left(\theta_{h}-\theta_{h}^{\ell}, y_{h}-y_{h}^{\ell+1}\right) & =\sum_{i=1}^{N_{h}}\left(\theta_{i}-\theta_{i}^{\ell}\right)\left(y_{i}-y_{i}^{\ell+1}\right) \\
& =\sum_{i=1}^{N_{h}} \mu_{i}^{-1}\left(-c^{-1} a\left(y_{h}-y_{h}^{\ell+1}, \psi_{i}\right)-\omega\left(y_{i}-y_{i}^{\ell+1}\right) \mu_{i}\right)\left(y_{i}-y_{i}^{\ell+1}\right) \\
& =\sum_{i=1}^{N_{h}} \mu_{i}^{-1}\left(-c^{-1} a\left(y_{h}-y_{h}^{\ell+1},\left(y_{i}-y_{i}^{\ell+1}\right) \psi_{i}\right)\right)-\omega \sum_{i=1}^{N_{h}}\left(y_{i}-y_{i}^{\ell+1}\right)^{2},
\end{aligned}
$$

where we have used (3.24). Secondly, we straightforwardly state the inequality

$$
a\left(y_{h}-y_{h}^{\ell+1},\left(y_{i}-y_{i}^{\ell+1}\right) \psi_{i}\right)=\left(y_{i}-y_{i}^{\ell+1}\right)^{2} a\left(\psi_{i}, \psi_{i}\right) \geq 0, \quad 1 \leq i \leq N_{h} .
$$

Thus, by applying this inequality to (3.25) we deduce

$$
\left(\theta_{h}-\theta_{h}^{\ell}, y_{h}-y_{h}^{\ell+1}\right) \leq-\mu_{N_{h}}^{-1} c^{-1} a\left(y_{h}-y_{h}^{\ell+1}, y_{h}-y_{h}^{\ell+1}\right)-\omega\left(y_{h}-y_{h}^{\ell+1}, y_{h}-y_{h}^{\ell+1}\right) .
$$

Moreover, by using the coerciveness of $a$ and the expression of the constant $c$, we get

$$
\left(\theta_{h}-\theta_{h}^{\ell}, y_{h}-y_{h}^{\ell+1}\right) \leq-2 \mu_{N_{h}}^{-1} \alpha \beta^{-1}\left|\frac{\mathrm{d}}{\mathrm{d} X}\left(y_{h}-y_{h}^{\ell+1}\right)\right|^{2}-\omega\left|y_{h}-y_{h}^{\ell+1}\right|^{2},
$$

where $|\cdot|$ is the usual norm in $L^{2}\left(L_{1}, L_{2}\right)$.

Next, we obtain a bound for $\lambda\left|\theta_{h}-\theta_{h}^{\ell+1}\right|^{2}$. For this, we first apply the contractivity, with constant $\lambda^{-1}$, of the Yosida operator $G_{\lambda}^{\omega}$ when $\lambda \omega=1 / 2$ and get:

$$
\begin{aligned}
\lambda\left|\theta_{h}-\theta_{h}^{\ell+1}\right|^{2} & \leq \lambda^{-1}\left|y_{h}+\lambda \theta_{h}-y_{h}^{\ell+1}-\lambda \theta_{h}^{\ell}\right|^{2} \\
& =\lambda^{-1}\left|y_{h}-y_{h}^{\ell+1}\right|^{2}+\lambda\left|\theta_{h}-\theta_{h}^{\ell}\right|^{2}+2\left(y_{h}-y_{h}^{\ell+1}, \theta_{h}-\theta_{h}^{\ell}\right) .
\end{aligned}
$$

Then, using (3.26) in the previous inequality, we deduce:

$$
\lambda\left|\theta_{h}-\theta_{h}^{\ell+1}\right|^{2} \leq \lambda^{-1}\left|y_{h}-y_{h}^{\ell+1}\right|^{2}+\lambda\left|\theta_{h}-\theta_{h}^{\ell}\right|^{2}-4 \mu_{N_{h}}^{-1} \alpha \beta^{-1}\left|\frac{\mathrm{d}}{\mathrm{d} X}\left(y_{h}-y_{h}^{\ell+1}\right)\right|^{2}-2 \omega\left|y_{h}-y_{h}^{\ell+1}\right|^{2} .
$$

So, taking into account the choice $\lambda \omega=1 / 2$ in (3.27), we can write:

$$
\lambda\left|\theta_{h}-\theta_{h}^{\ell+1}\right|^{2} \leq \lambda\left|\theta_{h}-\theta_{h}^{\ell}\right|^{2}-4 \mu_{N_{h}}^{-1} \alpha \beta^{-1}\left|\frac{\mathrm{d}}{\mathrm{d} X}\left(y_{h}-y_{h}^{\ell+1}\right)\right|^{2} \leq \lambda\left|\theta_{h}-\theta_{h}^{\ell}\right|^{2} .
$$


Therefore, the sequence $\left\{\left|\theta_{h}-\theta_{h}^{\ell}\right|^{2}\right\}$ is monotone decreasing and, so, convergent. Moreover, as we have

$$
0 \leq\left|\frac{\mathrm{d}}{\mathrm{d} X}\left(y_{h}-y_{h}^{\ell+1}\right)\right|^{2} \leq \frac{\mu_{N_{h}} \beta \lambda}{4 \alpha}\left(\left|\theta_{h}-\theta_{h}^{\ell}\right|^{2}-\left|\theta_{h}-\theta_{h}^{\ell+1}\right|^{2}\right)
$$

the sequence $\left\{y_{h}^{\ell+1}\right\}$ converges to $y_{h}$ as $\ell$ goes to infinity.

Remark 3.2. Although the convergence result is stated for the case $H=1$, numerical tests show good convergence properties for other choices of $H$. In these tests, it has also been observed that convergence does not deteriorate as the spatial mesh becomes finer.

\subsection{Optimization of the duality algorithm with functional parameters}

In this section, we analyze the optimal choice of the functional parameter $\omega$ in order to accelerate the convergence of the algorithm (3.7)-(3.8). This optimal choice is crucial to obtain a good practical performance of the algorithm as the number of iterations depends on $\omega$. For this purpose, notice that in the abstract notation (3.10)-(3.11), Yosida approximation of operator $G$ with parameter $1 /(2 \omega)$ is given by expression

$$
G_{1 / 2 \omega}^{\omega}(z)=2 \omega\left(I-\left(\frac{1}{2} I+\frac{1}{2 \omega} G\right)^{-1}\right)(z) .
$$

Next, we define the function $F_{\omega}: V \longrightarrow V$, given by:

$$
F_{\omega}(\theta)=G_{1 / 2 \omega}^{\omega}\left(y(\theta)+\frac{\theta}{2 \omega}\right)
$$

where, given $q \in V, y(q)$ is such that:

$$
A y(q)+c\left(B B^{*}\right)(\omega y(q))=f-c\left(B B^{*}\right)(q)
$$

Let $\bar{\theta}$ be a fixed point of $F_{\omega}$. Our aim is to accelerate the convergence of the fixed-point algorithm, by choosing $\omega$ such that:

$D F_{\omega}(\bar{\theta})$ being the Gâteaux-derivative of $F_{\omega}$ in $\bar{\theta}$.

$$
D F_{\omega}(\bar{\theta})=0
$$

Proposition 3.1. A sufficient condition for (3.29) is $\omega=2 y(\bar{\theta})$.

Proof. Let $z=y(\theta)+\frac{\theta}{2 \omega}$. Some straightforward calculations show that:

$$
\left\langle D F_{\omega}(\theta), q\right\rangle=\frac{\mathrm{d} G_{1 / 2 \omega}^{\omega}}{\mathrm{d} z}\left(y(\theta)+\frac{\theta}{2 \omega}\right)\left(y(q)+\frac{q}{2 \omega}\right), \quad \forall q \in V .
$$

If we can choose $\omega$ so that:

$$
\frac{\mathrm{d} G_{1 / 2 \omega}^{\omega}}{\mathrm{d} z}\left(y(\bar{\theta})+\frac{\bar{\theta}}{2 \omega}\right)=0
$$

then (3.29) is achieved. An easy application of the inverse function theorem leads to

$$
\frac{\mathrm{d} G_{1 / 2 \omega}^{\omega}}{\mathrm{d} z}(z)=2 \omega\left(1-\frac{1}{\frac{1}{2}+\frac{1}{2 \omega} G^{\prime}(t)}\right)(z)
$$

where $t$ verifies the equation

$$
\frac{1}{2} t+\frac{1}{2 \omega} G(t)=z
$$


TABLE 1. Number of iterations $\left(I_{d}\right)$ and quadratic error in Test 1.

\begin{tabular}{lcccc}
\hline & \multicolumn{2}{c}{$\omega=2$} & \multicolumn{2}{c}{$\omega=2 p$} \\
$\Delta x$ & $I_{d}$ & $e_{p}$ & $I_{d}$ & $e_{p}$ \\
\hline $10^{-2}$ & 7 & $1.2 \times 10^{-6}$ & 4 & $1.1 \times 10^{-6}$ \\
$10^{-3}$ & 7 & $1.2 \times 10^{-8}$ & 4 & $4.9 \times 10^{-9}$ \\
$10^{-4}$ & 7 & $7.7 \times 10^{-10}$ & 4 & $6.1 \times 10^{-10}$ \\
\hline
\end{tabular}

TABLE 2. Number of iterations $\left(I_{d}\right)$ for different $\omega$ in Test 1.

\begin{tabular}{cccccccc}
\hline$\omega$ & 0.02 & 0.2 & 1 & 2 & 3 & 20 & 200 \\
$I_{d}$ & 231 & 41 & 12 & 7 & 8 & 60 & 525 \\
\hline$\omega$ & $0.02 p$ & $0.2 p$ & $p$ & $2 p$ & $3 p$ & $20 p$ & $200 p$ \\
$I_{d}$ & 200 & 34 & 8 & 4 & 8 & 48 & 455 \\
\hline
\end{tabular}

provided that $z \neq 0$. So, using (3.30) and (3.31), a sufficient condition for (3.29) is:

$$
\frac{1}{2}+\frac{1}{2 \omega} G^{\prime}(t)=1, \quad \text { with } z=y(\bar{\theta})+\frac{\bar{\theta}}{2 \omega} \text { in }(3.32)
$$

which is equivalent to $\omega(X)=G^{\prime}(t)(X)$. Finally, as $\bar{\theta}=G(y(\bar{\theta}))-\omega y(\bar{\theta})$, it easily follows the equivalence:

$$
z=y(\bar{\theta})+\frac{\bar{\theta}}{2 \omega} \quad \Longleftrightarrow \quad t=y(\bar{\theta})
$$

and the optimal choice for the parameter is $\omega=2 y(\bar{\theta})$.

Remark 3.3. Notice that the optimal choice of the parameter depends on solution $y(\bar{\theta})$. So, when the exact solution is unknown, in our practical implementation $\omega=2 P_{m}$ is taken, $P_{m}$ being the approximation of solution in the last step of the characteristics loop.

\subsection{Some test examples}

In this section we present several tests that show the behaviour of the previously described numerical techniques and illustrate the related theoretical results.

Test 1. Let us consider the following nonlinear diffusion problem:

$$
\left\{\begin{array}{l}
-\frac{\mathrm{d}}{\mathrm{d} x}\left(h^{2} \frac{\mathrm{d} p}{\mathrm{~d} x}+h^{3} p \frac{\mathrm{d} p}{\mathrm{~d} x}\right)=f \quad \text { in }(0,1) \\
p(0)=p(1)=1
\end{array}\right.
$$

where $h(x)=2-x$ and $f$ is such that the solution is $p(x)=1+x-x^{2}$.

Table 1 shows the number of iterations, $I_{d}$, of the duality method (with optimal constant and variable parameters) and the relative quadratic error $e_{p}$ (between the numerical approximation and the analytical solution) for different mesh sizes and a tolerance equals to $10^{-7}$. Table 2 illustrates the optimality of parameters in terms of the number of iterations. The same kind of results have been observed in the case $h=1$. 
TABLE 3. Number of iterations for different parameter choices in Test 2.

\begin{tabular}{|c|c|c|c|c|c|c|}
\hline \multirow[b]{2}{*}{$\Delta x$} & \multicolumn{2}{|c|}{$\omega=2$} & \multicolumn{2}{|c|}{$\omega=2 p_{m}$} & \multicolumn{2}{|c|}{$\omega=2 p$} \\
\hline & $I_{c}$ & $\overline{I_{d}}$ & $I_{c}$ & $\overline{I_{d}}$ & $I_{c}$ & $\overline{I_{d}}$ \\
\hline $10^{-2}$ & 796 & 4.1 & 796 & 3.0 & 796 & 2.9 \\
\hline $10^{-3}$ & 6899 & 3.0 & 6899 & 2.6 & 6899 & 2.5 \\
\hline $10^{-4}$ & 58569 & 2.2 & 58569 & 2.3 & 58569 & 2.2 \\
\hline
\end{tabular}

TABLE 4. Number of iterations for different parameter choices in Test 3.

\begin{tabular}{crrrrrrr}
\hline & \multicolumn{2}{c}{$\omega=2$} & \multicolumn{2}{c}{$\omega=2 p_{m}$} & \multicolumn{2}{c}{$\omega=2 p$} \\
$\Delta x$ & \multicolumn{1}{c}{$I_{c}$} & $\overline{I_{d}}$ & & \multicolumn{1}{c}{$I_{c}$} & $\overline{I_{d}}$ & $I_{c}$ & $\overline{I_{d}}$ \\
\hline $10^{-2}$ & 1041 & 3.8 & & 1041 & 3.0 & 1041 & 2.8 \\
$10^{-3}$ & 9062 & 2.8 & & 9062 & 2.6 & 9062 & 2.5 \\
$10^{-4}$ & 76766 & 2.1 & & 76766 & 2.3 & 76766 & 2.1 \\
\hline
\end{tabular}

Test 2. Let us now consider the convection-diffusion problem which consists in finding the pressure, $p$, such that:

$$
\left\{\begin{array}{l}
10 \frac{\mathrm{d}}{\mathrm{d} x}(h p)-\frac{\mathrm{d}}{\mathrm{d} x}\left(h^{2} \frac{\mathrm{d} p}{\mathrm{~d} x}+h^{3} p \frac{\mathrm{d} p}{\mathrm{~d} x}\right)=f \quad \text { in }(0,1) \\
p(0)=p(1)=1
\end{array}\right.
$$

where $h(x)=2-x$ and $f$ is such that the solution is $p(x)=1+x-x^{2}$. We have taken $k=0.5 \Delta x$ as time step. The obtained results are shown in Table 3, where $I_{c}$ is the number of iterations of the characteristics algorithm and $\overline{I_{d}}$ is the average number of iterations in the duality algorithm per characteristics loop iteration. The tolerance has been taken to $10^{-10}$ both in the characteristics and the duality methods.

Test 3. Let us now consider the convection-diffusion problem which consists in finding the pressure, $p$, such that:

$$
\left\{\begin{array}{l}
10 \frac{\mathrm{d} p}{\mathrm{~d} x}-\frac{\mathrm{d}}{\mathrm{d} x}\left((1+p) \frac{\mathrm{d} p}{\mathrm{~d} x}\right)=f \quad \text { in }(0,1) \\
p(0)=p(1)=1
\end{array}\right.
$$

where $f$ is such that the solution is $p(x)=1+x-x^{2}$. Notice that this test corresponds to Test 2 with $h=1$. We have also taken $k=0.5 \Delta x$ as time step. The obtained results are shown in Table 4 and are very similar to those of Test 2. Notice that in the case $h=1$ convergence has been theoretically stated.

Test 4. In [17], the following compressible Reynolds equation is proposed to model lubricated rough surfaces:

$$
\begin{cases}300 \frac{\mathrm{d}}{\mathrm{d} x}(h p)-\frac{\mathrm{d}}{\mathrm{d} x}\left(0.4 h^{2} \frac{\mathrm{d} p}{\mathrm{~d} x}+h^{3} p \frac{\mathrm{d} p}{\mathrm{~d} x}\right)=0 & \text { in }(0,1) \\ p(0)=p(1)=1 & \end{cases}
$$

where $h(x)=2-x+0.6 \sin (100 \pi x)$. Table 5 shows the number of iterations obtained for constant and functional parameters for different mesh sizes and maximum relative error $\varepsilon=5 \times 10^{-9}$.

Analogous results to those in Table 2 have been obtained in Tests 2 to 4, the optimal parameters being the same as in Test 1. 
TABLE 5. Number of iterations in Test 4.

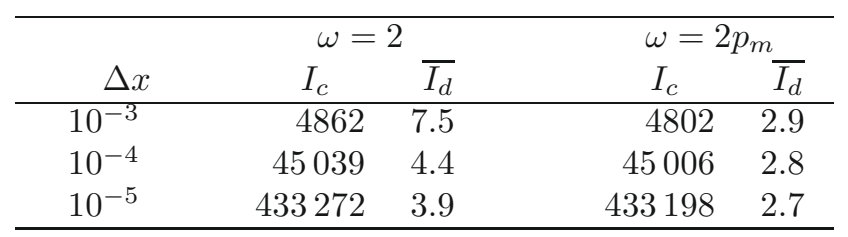

\section{Numerical SOlution of the Elastic PROBlem}

In this section we briefly describe the numerical methods to solve the elastic problem $\left(\mathcal{P}_{E}^{n+1}\right)$ for a given pressure approximation. For simplicity, we drop the index $n$ throughout this section. First, we propose a Hermite cubic finite element method (see Reddy [22], for example) for spatial discretization. For this, we consider the space

$$
W_{h}=\left\{w_{h} \in \mathcal{C}^{1}(0, L) /\left.w_{h}\right|_{E} \in \mathcal{P}_{3}, \forall E \in \tau_{h} ; w_{h}(0)=w_{h}(L)=w_{h}^{\prime}(0)=w_{h}^{\prime}(L)=0\right\},
$$

and the convex set associated to the unilateral constraint is approximated by

$$
K_{0 h}=\left\{w_{h} \in W_{h} / w_{h}\left(x_{i}\right) \geq \delta\left(x_{i}\right), \forall x_{i} \text { node of } \tau_{h}\right\} .
$$

Then, a variational inequality formulation of the discretized problem is posed as follows:

For $P_{h} \in V_{h}$ given, find $U_{h} \in K_{0 h}$ solution of:

$$
\begin{aligned}
\int_{0}^{L} \frac{\mathrm{d} U_{h}}{\mathrm{~d} X}\left(\frac{\mathrm{d} w_{h}}{\mathrm{~d} X}-\frac{\mathrm{d} U_{h}}{\mathrm{~d} X}\right) \mathrm{d} X+\eta \int_{0}^{L} \frac{\mathrm{d}^{2} U_{h}}{\mathrm{~d} X^{2}}\left(\frac{\mathrm{d}^{2} w_{h}}{\mathrm{~d} X^{2}}-\frac{\mathrm{d}^{2} U_{h}}{\mathrm{~d} X^{2}}\right) \mathrm{d} X \geq \\
K \int_{0}^{L}\left(P_{h}-1\right) \chi_{\left[L_{1}, L_{2}\right]}\left(w_{h}-U_{h}\right) \mathrm{d} X, \quad \forall w_{h} \in K_{0 h} .
\end{aligned}
$$

Among the different suitable methods for elliptic variational inequalities [15], we have compared the results obtained with projected Gauss-Seidel, projected gradient and Uzawa duality methods, this last one being the most efficient in terms of the number of iterations and CPU time. For completeness, we next briefly describe the application of this duality algorithm. Thus, we consider a 1-d finite element mesh with $N$ nodes of the interval $[0, L]$ and we note by $A$ and $b$ the matrix and the second member, respectively, of the system associated to the discretized problem. We denote by $u$ a vector of $2 N$ components that contains the value of the approximated solution (odd components) and its first derivative (even components) at the nodes. Thus, the unilateral constraint only acts on the odd components and the scheme of the algorithm can be written as follows:

1. Initialize the vector $\lambda^{0} \in \mathbb{R}_{+}^{2 N}$.

2. $k$-step: For $\lambda^{k}$ given, we compute $u^{k}$, such that:

$$
A u^{k}=b+\lambda^{k} .
$$

3. We apply a step of projected gradient for the dual maximization problem, i.e. for $u^{k}$ given we compute for $j=1, \ldots, N$ :

$$
\begin{aligned}
\lambda_{2 j-1}^{k+1} & =\max \left\{\lambda_{2 j-1}^{k}+\rho\left(\delta_{2 j-1}-u_{2 j-1}^{k}\right), 0\right\} \\
\lambda_{2 j}^{k+1} & =\lambda_{2 j}^{k}+\rho\left(\delta_{2 j}-u_{2 j}^{k}\right) .
\end{aligned}
$$


In the previous scheme, the parameter $\rho$ is associated to the gradient method and, for convergence purposes, it is chosen such that $\rho<\mu_{\text {min }}, \mu_{\text {min }}$ being the minimum eigenvalue of the symmetric positive definite matrix $A$ (see Ciarlet [9], for details). We have noted by $\delta_{2 j-1}$ the value of the obstacle at the node $j$ of the mesh.

\section{NumericAl SOlution OF THE COUPLED PROBlem}

In Section 2 we have described the fixed point algorithm to approximate the solution of the coupled problem, by sequentially solving the elastic and hydrodynamic subproblems until convergence. In order to validate this algorithm we have successfully performed several numerical tests with analytical solution, one of them being Test 5 in this section. Next, we present a test with real data in order to simulate an elastohydrodynamic process taking place in a real head-tape magnetic storage device.

Test 5: We consider the problem defined by equations:

$$
\begin{array}{ll}
\frac{\mathrm{d}}{\mathrm{d} X}(P H)-\frac{\mathrm{d}}{\mathrm{d} X}\left(\alpha H^{2} \frac{\mathrm{d} P}{\mathrm{~d} X}+\beta H^{3} P \frac{\mathrm{d} P}{\mathrm{~d} X}\right)=g & \text { in }\left(L_{1}, L_{2}\right), \\
-\frac{\mathrm{d}^{2} U}{\mathrm{~d} X^{2}}+\eta \frac{\mathrm{d}^{4} U}{\mathrm{~d} X^{4}}=K(P-1) \chi_{\left[L_{1}, L_{2}\right]}+f & \text { in }(0, L), \\
H=U-\delta & \text { in }(0, L), \\
P\left(L_{1}\right)=P\left(L_{2}\right)=1, & \\
U(0)=U(L)=\frac{\mathrm{d} U}{\mathrm{~d} X}(0)=\frac{\mathrm{d} U}{\mathrm{~d} X}(L)=0, &
\end{array}
$$

where we introduce the new functions $f$ and $g$ so that the exact solution is given by

$$
P(X)=\left(X-L_{1}\right)\left(L_{2}-X\right)+1, \quad U(X)=c X^{2}(X-L)^{2} .
$$

Moreover, we have chosen the values $c=21.11, L_{1}=0.2, L_{2}=0.8$ and $L=1$. Also, the parameters $\alpha, \beta, \eta$ and $K$ have been taken to the values

$$
\alpha=1.16 \times 10^{-2}, \quad \beta=3.04 \times 10^{-2}, \quad \eta=5.49 \times 10^{-4}, \quad K=3.0375 \times 10^{4},
$$

which are obtained by scaling a real device [6].

Concerning the data of the numerical methods, first we point out that all meshes have three regions with constant stepsize: a central one, over the head, and two other regions located at both sides of the head. Notice that the spatial mesh stepsize is much smaller in the central region than in the other two ones. The artificial time step is taken to $k=0.5 \Delta x$, where $\Delta x$ is the minimum spatial stepsize. Table 6 shows the pressure $\left(e_{P}\right)$ and displacement $\left(e_{U}\right)$ quadratic errors when using the duality algorithm with functional parameter $\omega=2 P_{m}$, $P_{m}$ being the computed solution in the last characteristics loop iteration. Moreover, $N_{l}, N_{c}$ and $N_{r}$ denote the number of nodes of the uniform meshes in the left, central and right regions of the domain for the elastic problem, $N_{c}$ also representing the number of mesh nodes for the domain $\left(L_{1}, L_{2}\right)$ in the Reynolds equation. For the numerical algorithms in the hydrodynamic problem a relative quadratic error stopping test equals to $10^{-7}$ has been considered. Moreover, in the elastic part, a direct heptadiagonal system solver has been used for the primal problem, $\rho=10^{-6}$ is taken in the iterations of the dual problem and a relative error stopping test of $10^{-6}$ is considered. Finally, the tolerance for the fixed point algorithm is $10^{-8}$ both for the pressure and the displacement, the number of iterations being noted by $I_{p}$ in Table 6 . Notice that as the exact displacement and pressure are known, we can calculate the relative quadratic error between approximated and exact solutions. In Table 6 we can observe the good behaviour in both cases, and the decrease of the errors as the number of nodes increases. 
TABLE 6. Quadratic errors for displacements and pressure in Test 5.

\begin{tabular}{cccc}
\hline$N_{l}+N_{c}+N_{r}$ & $e_{P}$ & $e_{U}$ & $I_{p}$ \\
\hline $20+501+20$ & $3.4 \times 10^{-9}$ & $4.05 \times 10^{-8}$ & 3 \\
$40+1001+40$ & $1.7 \times 10^{-9}$ & $9.90 \times 10^{-8}$ & 3 \\
$80+2001+80$ & $8.5 \times 10^{-10}$ & $4.20 \times 10^{-8}$ & 2 \\
\hline
\end{tabular}

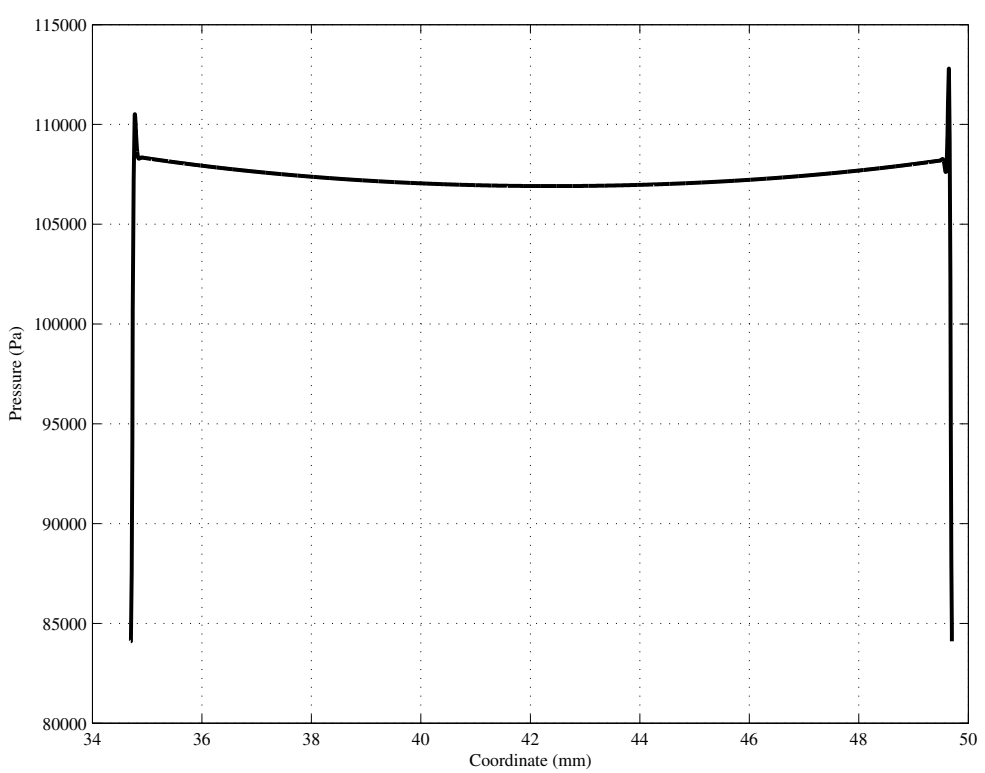

Figure 3. Computed pressure in Test 6 for a mesh with 12000 nodes in central region and 1000 nodes at both sides.

Test 6: We consider a real device characterized by the parameters detailed in (2.6). The dimensionless coefficients $\alpha, \beta, \eta$ and $K$ are the same as in Test 5 , as well as the scaled equations with $f=g=0$.

Among the different obtained results, in Figures 3 and 4 we present the computed pressure and headtape gap, respectively, for a particular set of parameters in the numerical methods. More precisely, they correspond to a mesh with 12000 nodes at the central region over the head and 1000 nodes at each side, while $k=0.5 \min \{\Delta x\}=6.25 \times 10^{-7}$ has been chosen. In Figure 3 we can appreciate the presence of sharp pressure spikes near both ends of the head, the one on the right being sharper. Also the gap function presents the typical spike near the outflow boundary. These results agree with those presented in the related literature (see [6,23], for example).

Finally, in Figures 5 and 6 we present the convergence behaviour of the pressure and gap, respectively, when mesh is refined. Notice the good behaviour despite the presence of spikes and the not so smooth pressure and gap in this real data elastohydrodynamic setting.

\section{Conclusions}

In this paper we have introduced new numerical methods to solve a coupled system governed by a nonlinear compressible Reynolds equation and a rod model. The system models the elastohydrodynamic process taking place in head-tape magnetic reading devices. After describing the involved numerical techniques, their good performance is well illustrated by their application to academic problems with closed form solution. The numerical 


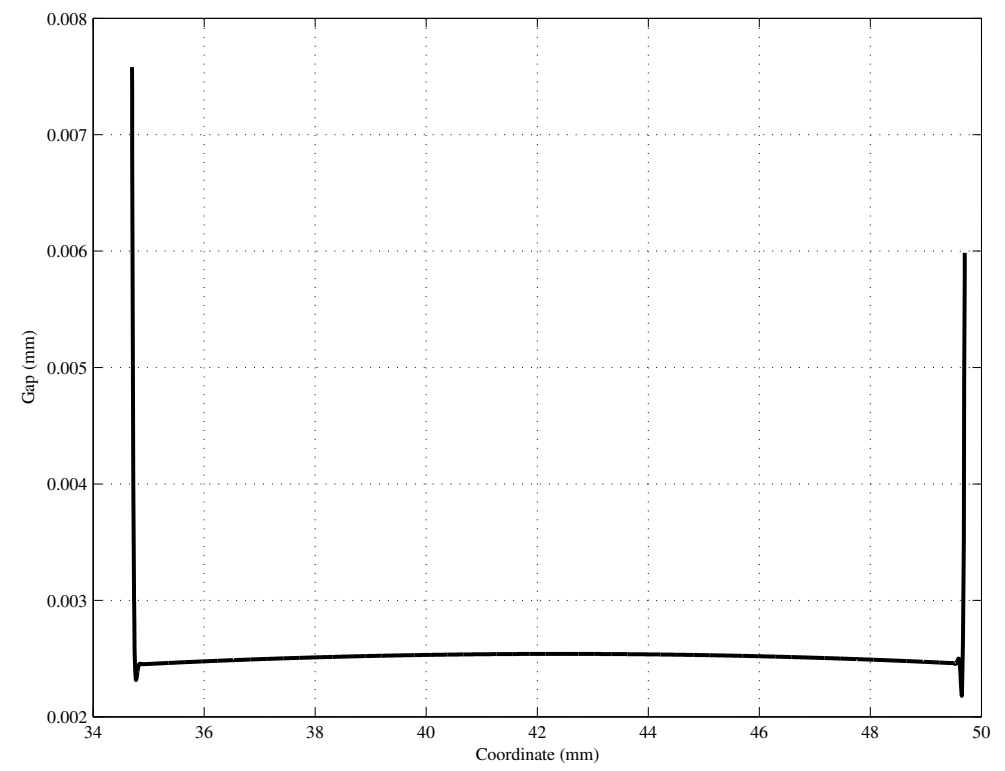

Figure 4. Computed gap in Test 6 for a mesh with 12000 nodes in central region and 1000 nodes at both sides.

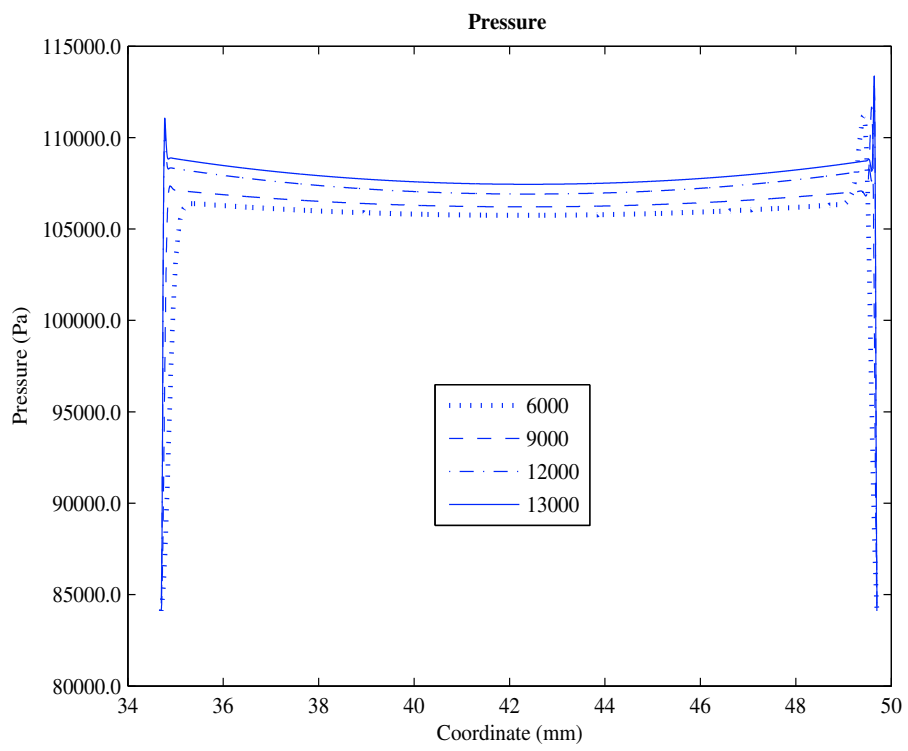

FiguRE 5. Computed pressure for meshes with different numbers of nodes in central region.

simulation of devices with real data sets is also carried out. The main original point of the numerical techniques is the use of a nonclassical duality method for solving the nonlinear diffusion term, jointly with a characteristics scheme for the convection dominating aspect. Concerning the duality method, which is based on Yosida regularization of maximal monotone operators, partial and interesting theoretical results about convergence have 


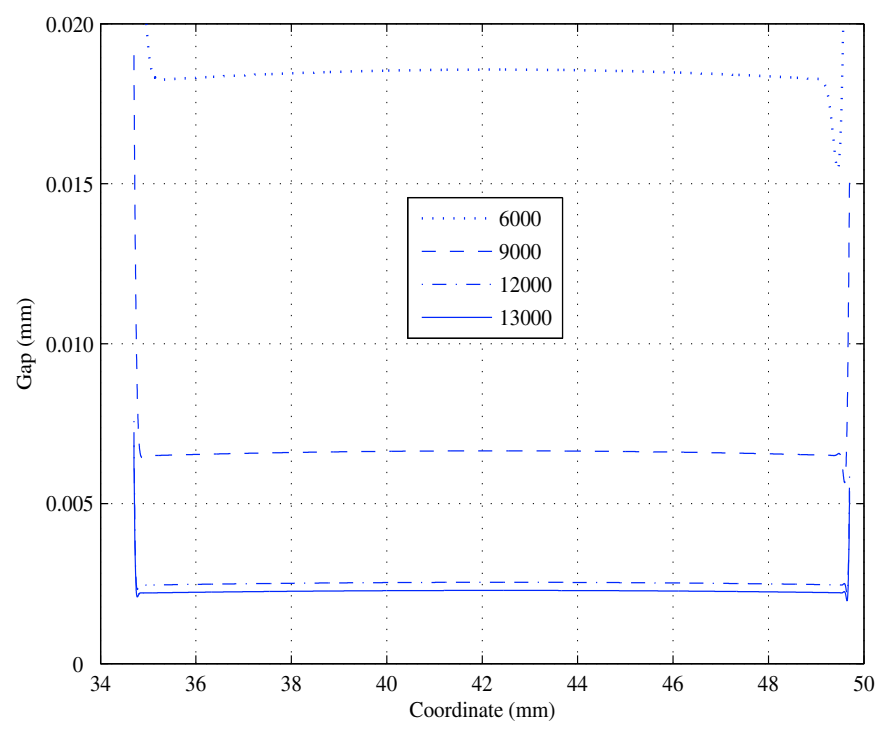

Figure 6. Computed gap for meshes with different numbers of nodes in central region.

been obtained in a new functional equation setting. Furthermore, when using functional parameters in the method, an optimal choice has been found. This aspect is relevant for saving computational costs and it has been illustrated accordingly by several test examples.

The extension to two spatial dimensions has also been studied by the authors, from the modelling and numerical point of view [3]. In fact, for example, the increasing use of smaller flow factors in recording requires narrower tapes, leading to side flow effects which are neglected in one dimensional approaches. Thus, an additional interest of the proposed numerical techniques for the first-order slip Reynolds equations is that they can be easily extended to two spatial dimensions. Also, in the 2-d setting, the fixed point iteration should uncouple the hydrodynamic equations and the appropriate elastic model. In [3] a new elastohydrodynamic Reynolds-Koiter model governing a head-tape magnetic reading device is proposed and the numerical methods here applied to the nonlinear compressible Reynolds equation are extended to the 2-d case. In order to take into account curvature effects, the tape movement is governed by a Koiter model and appropriate numerical methods are used.

Finally, in order to improve the accuracy of the methods in complex geometries, the use of multigrid or adaptive meshing is being studied by the authors. Both techniques are specially useful in two dimensions when a complex or discontinuous geometry of the head could lead to discontinuities or large gradients in the main unknowns. This is not the case in the present paper. In adaptive mesh refinement the pressure gradient could be considered as the metric for making subdivision decisions.

Acknowledgements. The authors wish to thank Ministerio de Educación y Ciencia (projects MTM2004-05796-C02-01 and MTM2007-67596-C02-01) and Xunta de Galicia (project PGIDIT05PXIC30302PN) for their financial support. Also the authors thank both anonymous referees for their comments which improved the final version of the paper. 


\section{REFERENCES}

[1] I. Arregui and C. Vázquez, Finite element solution of a Reynolds-Koiter coupled problem for the elastic journal bearing. Comput. Meth. Appl. Mech. Engrg. 190 (2001) 2051-2062.

[2] I. Arregui, J.J. Cendán and C. Vázquez, A duality method for the compressible Reynolds equation. Application to simulation of read/write processes in magnetic storage devices. J. Comput. Appl. Math. 175 (2005) 31-40.

[3] I. Arregui, J.J. Cendán and C. Vázquez, Numerical simulation of head-tape magnetic reading devices by a new two dimensional model. Finite Elem. Anal. Des. 43 (2007) 311-320.

[4] A. Bermúdez, Un método numérico para la resolución de ecuaciones con varios términos no lineales. Aplicación a un problema de flujo de gas en un conducto. Rev. R. Acad. Cienc. Exactas Fís. Nat. 78 (1981) 89-96.

[5] A. Bermúdez and C. Moreno, Duality methods for solving variational inequalities. Comput. Math. Appl. 7 (1981) 43-58.

[6] B. Bhushan, Tribology and Mechanics of Magnetic Storage Devices. Springer, New York (1996).

[7] G. Buscaglia and M. Jai, A new numerical scheme for non uniform homogenized problems: application to the nonlinear Reynolds compressible equation. Math. Probl. Engrg. 7 (2001) 355-378.

[8] G. Buscaglia, S. Ciuperca and M. Jai, Existence and uniqueness for several nonlinear elliptic problems arising in lubrication theory. J. Diff. Eq. 1 (2005) 187-215.

[9] P.G. Ciarlet, Introduction à l'Analyse Numérique Matricielle et à l'Optimisation. Masson, Paris (1982).

[10] J. Durany, G. García and C. Vázquez, Numerical computation of free boundary problems in elastohydrodynamic lubrication. Appl. Math. Modelling 20 (1996) 104-113.

[11] J. Durany, G. García and C. Vázquez, Simulation of a lubricated Hertzian contact problem under imposed load. Finite Elem. Anal. Des. 38 (2002) 645-658.

[12] A. Friedman, Mathematics in Industrial Problems, IMA 97. Springer, New York (1994).

[13] A. Friedman and B. Hu, Head-media interaction in magnetic recording, Arch. Rational Mech. Anal. 140 (1997) 79-101.

[14] A. Friedman and J.I. Tello, Head-media interaction in magnetic recording. J. Diff. Eq. 171 (2001) 443-461.

[15] R. Glowinski, J.L. Lions and R. Trémolières, Analyse Numérique des Inéquations Variationnelles. Dunod, Paris (1976).

[16] J. Heinrich and S. Wadhwa, Analysis of self-acting bearings: a finite element approach. Tribol. Mech. Magnet. Stor. Syst., STLE SP-21 3 (1986) 152-159.

[17] M. Jai, Homogenization and two-scale convergence of the compressible Reynolds lubrification equation modelling the flying characteristics of a rough magnetic head over a rough rigid-disk surface. RAIRO Modél. Math. Anal. Numér. 29 (1995) 199-233.

[18] C.A. Lacey and F.E. Talke, A tightly coupled numerical foil bearing solution. IEEE Trans. Magn. 26 (1990) 3039-3043.

[19] C.A. Lacey and F.E. Talke, Measurement and simulation of partial contact at the head-tape interface. ASME J. Tribol. 114 (1992) 646-652.

[20] C. Parés, J. Macías and M. Castro, Duality methods with an automatic choice of parameters. Application to shallow water equations in conservative form. Numer. Math. 89 (2001) 161-189.

[21] C. Parés, J. Macías and M. Castro, On the convergence of the Bermúdez-Moreno algorithm with constant parameters. Numer. Math. 92 (2002) 113-128.

[22] J.N. Reddy, An Introduction to Finite Element Methods. McGraw-Hill (1993).

[23] K.J. Stahl, J.W. White and K.L. Deckert, Dynamic response of self acting foil bearings. IBM J. Res. Dev. 18 (1974) $513-520$.

[24] S. Tan and F.E. Talke, Numerical and experimental investigations of head-tape interface in a digital linear tape drive. ASME J. Tribol. 123 (2001) 343-349.

[25] S.R. Wu and J.T. Oden, A note on some mathematical studies in elastohydrodynamic lubrication. Int. J. Eng. Sci. 25 (1987) 681-690.

[26] Y. Wu and F.E. Talke, Design of a head-tape interface for ultra low flying. IEEE Trans. Magn. 32 (1996) $160-165$.

[27] Y. Wu and F.E. Talke, Finite element based head-tape interface simulation including head-tape surface asperity contacts. IEEE Trans. Magn. 34 (1998) 1783-1785.

[28] Y. Wu and F.E. Talke, A finite element simulation of the two-dimensional head-tape interface for head contours with longitudinal bleed slots. Tribol. Int. 22 (2000) 123-130. 